\title{
Molecular epidemiology of human and animal tuberculosis in Ibadan, Southwestern Nigeria
}

\author{
A.O. Jenkins ${ }^{a, *}$, S.I.B. Cadmus ${ }^{b}$, E.H. Venter ${ }^{a}$, C. Pourcel $^{c}$, Y. Hauk $^{c}$, G. Vergnaud $^{c, d}$, \\ J. Godfroid ${ }^{a, e}$ \\ ${ }^{a}$ Department of Veterinary Tropical Diseases, Faculty of Veterinary Science, University of Pretoria, Onderstepoort, 0110 \\ Pretoria, South Africa \\ ${ }^{\mathrm{b}}$ Department of Veterinary Public Health and Preventive Medicine, University of Ibadan, Ibadan, Nigeria \\ c Institut de Génétique et Microbiologie Bât 400, Université Paris-Sud 91405, Orsay cedex, France \\ d DGA/MRIS- Mission pour la Recherche et l'Innovation Scientifique, 92220 Bagneux, France \\ ${ }^{e}$ Department of Food Safety and Infection Biology, Norwegian School of Veterinary Science, Stakkevollveien 23, 9010 Troms $\varnothing$, \\ Norway
}

\begin{abstract}
From 2005 to 2007, Mycobacterium tuberculosis complex (MTC) strains were isolated from cattle, goats and pigs samples collected at the Bodija abattoir and from human samples from tuberculosis patients and livestock traders at the Akinyele cattle market in Ibadan, Southwestern Nigeria. Seventy four isolates obtained from humans (24) and livestock (50) were identified as MTC strains. Thirty two isolates were spoligotyped. Nineteen of these 32 isolates were identified as M. tuberculosis whilst 13 were identified as Mycobacterium bovis. M. bovis was isolated from two humans, whereas M. tuberculosis was isolated from a bovine, a pig and a goat. All the M. bovis isolates identified in this study belonged to the Africa 1 clonal complex. Multiple locus VNTR [variable number of tandem repeats] analysis (MLVA) was carried out on the 74 isolates. Three major clusters were defined. Group A consisted of $24 \mathrm{M}$. tuberculosis isolates (MLVA genotypes 1-18). One strain was isolated from a bovine and one from a pig. Group B consisted of $49 \mathrm{M}$. bovis strains (MLVA genotypes 19-48), mainly of cattle origin but also included four goat, nine pig and two human isolates. Group C consisted of a single M. tuberculosis isolate (MLVA genotype 49) obtained from a goat. Spoligotyping and MLVA confirmed it as clustering with the East Africa Indian clade found in humans in Sudan and the Republic of Djibouti. The isolation of three M. tuberculosis strains from livestock raises the question of their epidemiological importance as a source of infection for humans.
\end{abstract}

Keywords: Mycobacterium tuberculosis complex; Spoligotyping; MLVA; Zoonoses; Nigeria

\section{Introduction}

The Mycobacterium tuberculosis complex (MTC) includes mycobacterial species that are causative agents of human and animal tuberculosis. It includes M. tuberculosis, Mycobacterium bovis, Mycobacterium africanum, Mycobacterium microti, Mycobacterium canettii, Mycobacterium caprae and Mycobacterium pinnipedii. Bovine tuberculosis (BTB, caused by M. bovis) like human tuberculosis (TB, caused by $M$. tuberculosis) is a disease characterized by progressive development of specific granulomatous lesions or tubercles in lung tissue, lymph nodes or other organs. Bovine tuberculosis is a disease of nearly all warm-blooded animals, including man, with the highest risk groups being individuals with concomitant HIV/AIDS infection (Ayele et al., 2004). Bovine tuberculosis is thus a zoonotic disease and it can spread to humans through inhalation of infectious droplets as well as by ingestion of infected raw milk. Tuberculosis caused by M. tuberculosis has also been reported in animals that live in close proximity to humans (Ocepek et al., 2005; Cadmus et al., 2006; Srivastava et al., 2008).

The incidence of zoonotic BTB in Nigeria is also of great concern as its degree of transmission is unknown despite reports of isolation of $\mathrm{M}$. bovis in humans (Cadmus et al., 2006). In the light of this disease as a re-emerging zoonosis worldwide and particularly in developing countries like Nigeria, due to increasing incidences of HIV/AIDS infections (Salami and Katibi, 2006, 2007), a thorough epidemiological assessment is required. Previous epidemiological studies on human tuberculosis in Nigeria were based on mycobacterial culture and they revealed approximately $3.9 \%$ of culture positive isolates were M. bovis (Idigbe et al., 1986; Mawak et al., 2006). Recently, MTC strains isolated from human samples in Nigeria were characterized using molecular methods like spoligotyping. It was shown that $\mathrm{M}$. bovis was isolated from $5 \%$ of human isolates in Ibadan (Cadmus et al., 2006). Furthermore, spoligotype profiles of some M. bovis strains isolated in Nigeria had been previously reported by earlier

* Corresponding author. Tel.: +27 12529 8382; fax: +27 12529 8312.E-mail addresses: akinjenks@gmail.com, s27421474@tuks.co.za (A.O. Jenkins). 
studies in Cameroon (Njanpop-Lafourcade et al., 2001). In Mali, a strain with a similar spoligopattern to SB0944, reported previously in Nigeria, Chad and Cameroon was also observed in 13 of the 20 strains studied (Muller et al., 2008). This therefore gives strong indications regarding clonal distribution of M. bovis strains in West Africa (Muller et al., 2009), and further emphasizes the relevance of molecular techniques in the epidemiology of tuberculosis.

Interactions at cattle markets and abattoirs between different species of livestock, introduction of foreign breeds of cattle into Nigeria for trade purposes coupled with human to livestock interactions throughout the entire animal husbandry systems and trade are some predisposing factors which may be capable of enhancing transmission of MTC especially in a region where only passive tuberculosis surveillance is implemented.

In the last 20 years, DNA fingerprinting techniques have been developed and used to study the inter-individual transmission of MTC and other mycobacterial infections and they have facilitated epidemiological studies at a population level as well as in the species identification of rarely encountered mycobacteria (Van Soolingen, 2001). Spoligotyping is often used in molecular epidemiology of MTC as well as in species and strain differentiation. This technique highlights differences between species/strains due to loss of spacers at the direct repeat (DR) region in MTC (Kamerbeek et al., 1997). This mode of fingerprinting is also marked by a system of nomenclature and strain data capture and identification and it is particularly relevant for phylogeography. Another molecular method named multiple locus VNTR [variable number of tandem repeats] analysis (MLVA), also referred to as mycobacterial interspersed repetitive unit-variable number of tandem repeats (MIRU-VNTR) (Allix et al., 2006) is increasingly used. MLVA highlights strain differences indicated by allelic variation at these multiple loci unlike spoligotyping which highlights spacer differences at only one locus, the DR region. It has been used in developed countries for M. bovis transmission studies and has produced highly informative data when combined with traditional epidemiology (Allix et al., 2006). It has also been used in a number of African countries where it revealed very interesting information regarding the clonal expansion of M. bovis in West Africa (Muller et al., 2009). Using this technique in Africa will help in elucidating the extent of spread of MTC and addressing speculations regarding risk factors. It will help the regulatory bodies to highlight the essence of proper disease control when the impact has been emphasized.

This study aims at elucidating the impact of zoonotic BTB by utilizing spoligotyping and MLVA as epidemiological tools in the detection of zoonotic BTB in Ibadan and it follows as an extension from a previous study by Cadmus et al. (2006). Ibadan with a human population of approximately three million people is peculiar in Nigeria as a node for cattle trade between the northern and southern parts and a distributor of meat to several south western states. It presents with an epidemiologically relevant scenario in which there is a classical interaction between cattle, small ruminants and humans for prolonged periods, especially at abattoirs and cattle markets. In this study, a selected number of MTC isolates were spoligotyped. Furthermore, MLVA using 16 VNTR which were selected from 21 loci (Le Fléche et al., 2002), was carried out on the MTC isolates obtained from livestock tissues from the abattoir and human samples from the University College Hospital, Ibadan. These 16 VNTR loci were previously used in a pilot genotyping of $\mathrm{M}$. bovis isolates obtained from Belgium for which they showed moderately to highly discriminatory power (Jenkins et al., 2010).

\section{Materials and methods}

\subsection{Study location}

The study location, Ibadan, the capital of Oyo State, is the third largest city in Nigeria by population (after Lagos and Kano), and has the largest geographical area. It is located in Southwestern Nigeria, $126 \mathrm{~km}$ inland from Lagos and is a prominent transit point between the coastal region and the areas to the North of the country where cattle are reared.

\subsection{Samples}

Seventy-four strains of human and animal origin collected in Ibadan (24 human isolates and 50 isolates of animal origin) were analysed in this study. Human samples were collected from patients attending designated "Directly Observed Therapy" centers in Ibadan (University Hospital College, Jaja Clinic, Jericho Chest Hospital). Sputum samples were also obtained from livestock traders in the Akinyele cattle market. The Akinyele cattle market is the main market for receiving cattle and other livestock in Ibadan and it is about $19 \mathrm{~km}$ from Bodija abattoir. The human samples consisted of respiratory aspirates (obtained from adult patients with pulmonary tuberculosis (PTB) and HIV/AIDS patients), infant faeces and sputum.

About two thirds of cattle in Ibadan are slaughtered at the Bodija abattoir where sampling based on the detection of gross lesions compatible with BTB was done. Liver, kidneys and the draining lymph nodes of the pleural cavity and the associated lymph nodes of the head and oral cavity were sampled. These samples were obtained from pigs, cattle and goats during the years 2005-2007. 


\subsection{Sample processing, culture and DNA extraction}

The processing of lesions and sputum samples was based on the Becton Dickinson digestion and decontamination procedure and it was carried out for processing both the sputum and cattle samples (for the cattle samples, grinding with pestle and mortar was first done with the addition of sterile distilled water before the procedure) as described by Cadmus et al. (2006). The suspension obtained after sample processing was inoculated onto Lowenstein-Jensen slopes with pyruvate and/or glycerol and incubated at $37^{\circ} \mathrm{C}$ for between eight and 12 weeks. The culture positive samples were further subjected to smear microscopy using Ziehl Neelsen (ZN) stain. Acid fast bacilli were harvested for molecular typing analysis by scraping the growth from a slope into $200 \mathrm{~m} \mathrm{I} \mathrm{sterile} \mathrm{distilled} \mathrm{water} \mathrm{and} \mathrm{heating} \mathrm{at} 80^{\circ} \mathrm{C}$ for $1 \mathrm{~h}$.

\subsection{Spoligotyping}

Thirty two of the 74 isolates which were earlier identified by deletion analysis according to Warren et al. (2006) were spoligotyped. Nineteen of these 32 isolates were identified as M. tuberculosis whilst 13 were identified as $M$. bovis. Spoligotyping was done according to a standardized international method described by Kamerbeek et al. (1997) using a commercially available kit (Isogen Biosciences BV, Maarsen, The Netherlands). M. tuberculosis H37Rv, M. bovis BCG and sterile distilled water were used as controls. New spoligopatterns were submitted to the $M$. bovis spoligotype database (www.mbovis.org), and a new SB code (Spoligotype code) was assigned accordingly.

\subsection{Multiple locus variable (number of tandem repeats) analysis}

The MLVA was carried out on 74 isolates. The isolates were amplified using primers targeting 16 MLVA loci [ETRA, ETRB, ETRC, ETRD (alias MIRU4), ETRE (alias MIRU31), MIRU10, MIRU27, MIRU40, Mtub02, Mtub12, Mtub21, Mtub29, Mtub30, Mtub38, Mtub39 and QUB11A] previously used in a pilot study and were selected from a panel of 21 loci described by Le Fleche et al. (2002). PCR amplification was performed as previously described (Le Fleche et al., 2002). PCRs were performed in a total volume of $25 \mu$ l containing $2 \mu$ l of the DNA, $12.5 \mu$ l of $2 x$ Fermentas PCR Mastermix, $9.5 \mu$ l of RNA free water and $0.5 \mu$ l (20 pM) of each flanking primer. After an initial denaturation step at $94^{\circ} \mathrm{C}$ for $5 \mathrm{~min}$, the PCR cycles were $94{ }^{\circ} \mathrm{C}$ for $30 \mathrm{~s}, 62{ }^{\circ} \mathrm{C}$ for $1 \mathrm{~min}$ and $72{ }^{\circ} \mathrm{C}$ for $1.5 \mathrm{~min}$ (40 cycles). A final elongation step of $72{ }^{\circ} \mathrm{C}$ for $10 \mathrm{~min}$ and then hold at $4{ }^{\circ} \mathrm{C}$ till samples were electrophoresed on an agarose gel. The PCR products were loaded in the standard MLVA format on $2 \%$ agarose gels for most loci and 3\% agarose gels for Mtub02 and Mtub12 as previously described (Le Fleche et al., 2002). Allele sizes were estimated using a 100 bp plus ladder (Fermentas) as a size marker. Copy numbers were visually analysed from gel images by two independent readers.

\section{Results}

\subsection{Spoligotyping}

Spoligotyping confirmed the species identification of 19 M. tuberculosis and 13 M. bovis isolates. M. tuberculosis was isolated from a pig (p44), a goat (g49) and a bovine (c15). The predominant spoligotype amongst the M. tuberculosis isolates (68\%) belonged to the Cameroon (CAM) family also called LAM10-CAM in the SpolDB4 database. They had the SpolDB4 types 61,403 or 838. Some CAM variants lacked one or several additional spacers as seen in isolates h116, N115, h110. Loss of spacer 11 was common to these CAM variants. None of these CAM variant spoligotypes were found in the spolDB4 database and were defined as such based on common spacer deletions they share with the CAM family (Table 1a). All M. tuberculosis strains from PTB patients that were spoligotyped had identical SpolDB4 type 61 belonging to the CAM family. Furthermore, one of the SpolDB4 type 61 was isolated from a pig (p44). The other six spoligopatterns identified were from the poorly defined T, $\mathrm{H}$ and $\mathrm{U}$ families and interestingly one East African Indian (EAI) clade. One cattle isolate had the SpolDB4 type 53 from the T1 family whilst the SpolDB4 type 342 EAI was isolated from a goat (Table 1a).

Two of the $M$. bovis spoligopatterns were neither found in the $M$. bovis nor the spolDB4 databases, and have since been submitted. These spoligopatterns designated as SB1432 and SB1472 were a human (hb74) and a cattle (c20) isolate, respectively. Another human isolate (NB83) was identified as $M$. bovis and it shared an identical spoligopattern (SB0944) with 4 other $M$. bovis strains (c24, p36, c34, c3). The other isolates were of different spoligotypes which had been previously isolated in Nigeria and subsequently assigned SB numbers in the www.mbo-vis database. SB0951 spoligotype was isolated from both a cattle and a pig (Table 1b). One cattle isolate (c6) presented with SB1105 spoligotype which was first isolated from a dromedary in Chad (www.mbovis.org). Four spoligotypes predominantly from Nigerian cattle which were found in the www.mbovis database (SB951, SB1027, SB1105 and SB1025), are not found in the spolDB4 database. 
Table 1a

Spoligopattern of $19 \mathrm{M}$. tuberculosis isolates from humans and livestock in Ibadan.

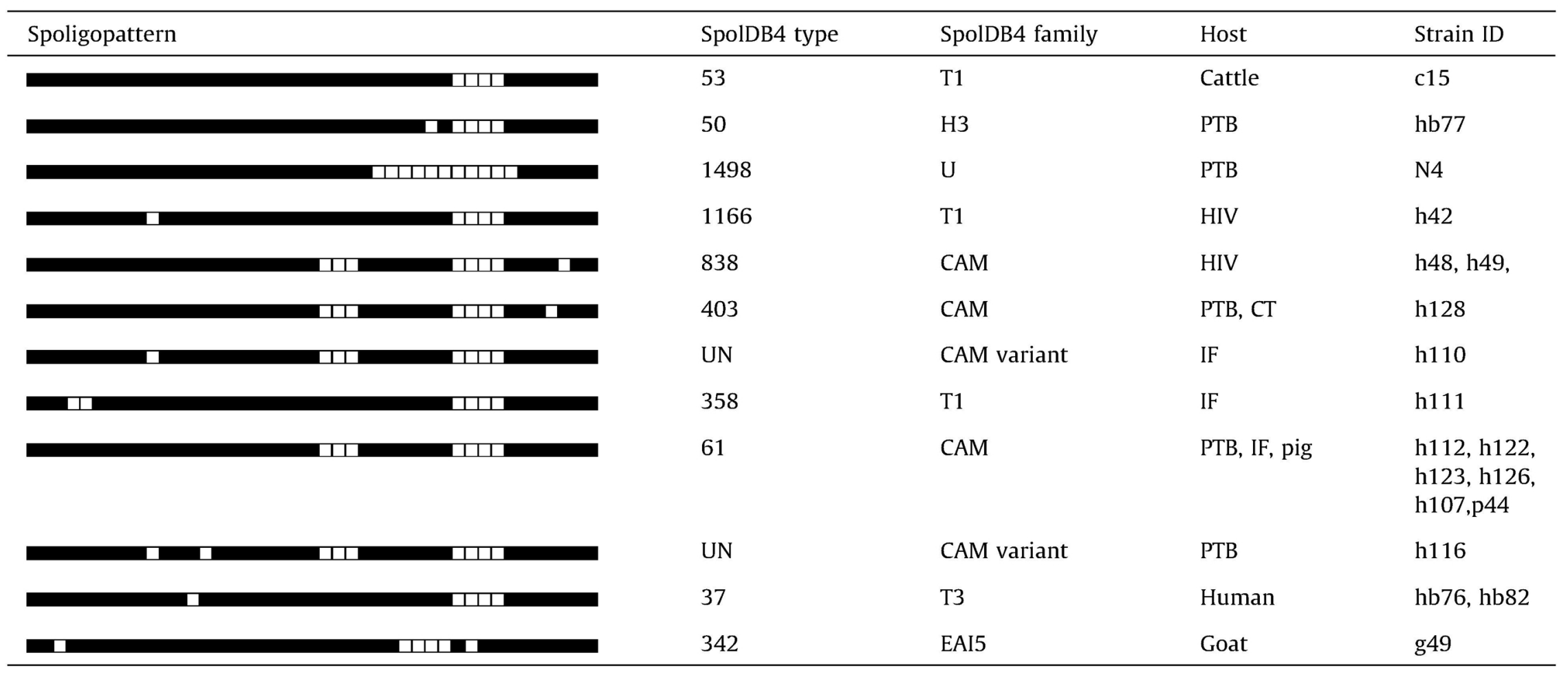

PTB (pulmonary tuberculosis patient); CT (cattle trader); IF (infant faeces). UN: spoligopatterns yet to be reported on the SpolDB4 database, spacer deletions however suggest that it is a CAM variant. 
Table 1b

Spoligopattern of $13 \mathrm{M}$. bovis isolates from humans and livestock in Ibadan.

\begin{tabular}{|c|c|c|c|c|}
\hline Spoligopattern & SB number & SpolDB4 family & Host & Strain ID \\
\hline لمسلب & SB1432 & UN & Human & NB83 \\
\hline पाता & SB1027 & UN & Cattle & c2 \\
\hline מחד & SB0944 & BOVIS1 & Cattle, pig, human & $\begin{array}{l}\text { c3, c24, } \\
\text { c34, p36, } \\
\text { hb74 }\end{array}$ \\
\hline שחسחسחדسחי & SB1473 & UN & Cattle & $\mathrm{c} 4$ \\
\hline صلाप & SB1105 & UN & Cattle & c6 \\
\hline 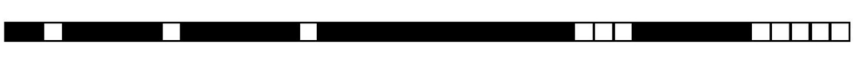 & SB1025 & UN & Cattle & c17 \\
\hline מחד & SB0951 & UN & Cattle, pig & c18, p35 \\
\hline صाप & SB1472 & UN & Cattle & $\mathrm{c} 20$ \\
\hline
\end{tabular}

UN: spoligopatterns not reported on the SpolDB4 database. 
Table 2

Genotypic and allelic diversity of the 16 MLVA loci of $M$. tuberculosis and M. bovis isolates from humans and livestock in Ibadan.

\begin{tabular}{|c|c|c|c|c|}
\hline \multirow[t]{2}{*}{ Locus } & \multicolumn{2}{|l|}{ M. bovis $n=49$} & \multicolumn{2}{|l|}{ M. tuberculosis $n=25$} \\
\hline & Number of alleles & Allelic diversity & Number of alleles & Allelic diversity \\
\hline ETRA-2165 & 4 & 0.29 & 5 & 0.603 \\
\hline ETRB-2461 & 4 & 0.628 & 4 & 0.297 \\
\hline ETRC-0577 & 5 & 0.39 & 4 & 0.497 \\
\hline ETRD-0580 & 3 & 0.192 & 3 & 0.157 \\
\hline ETRE-3192 & 4 & 0.581 & 3 & 0.41 \\
\hline MIRU10-0959 & 1 & 0 & 4 & 0.47 \\
\hline MIRU27-3006 & 3 & 0.429 & 3 & 0.46 \\
\hline MIRU40-0802 & 4 & 0.442 & 5 & 0.723 \\
\hline Mtub21-1955 & 2 & 0.153 & 2 & 0.08 \\
\hline Mtub30-2401 & 3 & 0.224 & 3 & 0.477 \\
\hline Mtub39-3690 & 3 & 0.081 & 7 & 0.823 \\
\hline Mtub02-0079 & 2 & 0.474 & 6 & 0.62 \\
\hline Mtub12-1121 & 1 & 0 & 1 & 0 \\
\hline Mtub29-2347 & 4 & 0.412 & 3 & 0.62 \\
\hline Mtub38-3663 & 2 & 0.041 & 2 & 0.153 \\
\hline QUB11A-2163 & 8 & 0.456 & 7 & 0.72 \\
\hline Locus & Total number of genotypes & Genotypic index & Total number of genotypes & Genotypic index \\
\hline MLVA 16 & 30 & 0.969 & 19 & 0.97 \\
\hline
\end{tabular}


Allelic diversity was calculated using Simpson's diversity index (Hunter and Gaston, 1988). For the M. bovis strains, nine loci were regarded as highly discriminatory in this assay i.e. $h>0.30$, whilst three loci (ETRD, Mtub21 and Mtub30) are moderately discriminatory i.e. $0.3>h>0.20$, whilst one locus (Mtub38) provided very little polymorphism and had the value $h=0.041$. Two loci (MIRU10 and Mtub12) were not polymorphic in this population (Table 2). The diversity index for the MLVA assay used here was 0.969 . The allelic diversity values for the $M$. tuberculosis isolates revealed 10 highly polymorphic loci $(h>0.30)$ whilst two loci, ETRD and Mtub38 were moderately polymorphic $(0.3>h>0.20)$. Only one locus (Mtub12) was monomorphic with these $M$. tuberculosis strains. The genotypic index for the 16 loci panel for the M. tuberculosis was 0.97 (Table 2).

The MLVA was carried out on 74 isolates by amplifying 16 MLVA loci. The obtained copy numbers were analysed using BioNumerics software. Absence of PCR amplification at some loci, presumably for technical reasons rather than the unlikely absence of the target locus was treated as absence of data (and not the " 0 " state). In the dendogram which was drawn by UPGMA (Fig. 1), five MLVA loci provided $100 \%$ typability. However, MIRU10 failed to amplify with 14 isolates, hence only $81 \%$ typability and subsequently no copy number was assigned to these isolates at this locus. Ten of these isolates were M. tuberculosis and were isolated from PTB patients.

Three major cluster groups, A, B and C were defined in the dendogram (Fig. 1). Group A isolates (MLVA genotypes 1-18) were all M. tuberculosis isolates $(n=24)$ and were further divided into two subgroups: Ai (MLVA genotypes $1-8)$ and Aii (MLVA genotypes 9-18). All the isolates in group A are members of the modern clade of M.tuberculosis with Ai comprising different spoligotypes of the $\mathrm{T}, \mathrm{H}$, and $\mathrm{U}$ families, whilst Aii strains were all of the CAM family. Group Ai consists of eight isolates: six human, one cattle and one pig isolates. The group Aii was divided into two sub groups, group Aii-a (MLVA genotypes 9-13) which consists of eight strains; four strains isolated from infant faeces, three strains from patients diagnosed with pulmonary tuberculosis and one strain from an HIV patient, whilst Aii-b (MLVA genotypes 14-18) also consists of eight strains, seven of which were isolated from patients diagnosed with pulmonary tuberculosis and one strain from an HIV patient.

All the isolates in group B were M. bovis $(n=49)$ and were predominantly of cattle origin. There were however four goat, nine pig and two human isolates (hb74 and NB83). The spoligotype of the human M. bovis strain hb74 is SB0944 and has been previously described in cattle in Nigeria, Cameroon, Chad and Mali. The strain NB83 with spoligotype SB1432 has also been previously described in cattle in Nigeria (Cadmus et al., 2006) but not in humans. Group B consisting of 49 M. bovis isolates was resolved into 30 MLVA genotypes (MLVA genotypes 19-48) and further divided into eight subgroups. MLVA genotype 21 was found in six strains of cattle origin. Only one of the six strains (c24) was spoligotyped and it had the same SB0944 spoligopattern as the human M. bovis isolate hb94. The closely related MLVA genotype 19 was also identified in three pig isolates (p36, p37 and p38). MLVA genotype 28 was common to strains isolated from different species of animals: g47 was isolated from a goat whereas c59, c60 and c63 were isolated from cattle. Four other strains showed MLVA genotype 30 and were isolated from pigs (p41, p43 and p45) and a bovine (b3) (Fig. 1). Group C consisted of a single M. tuberculosis isolate obtained from a goat. Spoligotyping confirmed it as having the pattern EAI5 (Table 1a).

\section{Discussion}

In the present report, 74 isolates obtained from humans (24) and livestock (50) in Ibadan, a confluence city in Southwestern Nigeria were analysed using spoligotyping and MLVA. Interestingly, M. bovis was isolated from two humans, whereas M. tuberculosis strains were isolated from three animals, a bovine, a pig and a goat. The primary reason for screening human samples in this study was to identify zoonotic tuberculosis due to M. bovis. The two human M. bovis isolates (hb74 and NB83) had clearly distinct spoligopatterns and MLVA genotypes, suggesting that they originated from different sources. The spoligotype of strain NB83 is identical to SB1432 in the www.mbovis.org database whilst hb74 has spoligotype SB0944 which has been previously reported in cattle in Nigeria (Cadmus et al., 2006), Chad, Cameroon (Njanpop-Lafourcade et al., 2001), Mali (Muller et al., 2008), France (Haddad et al., 2001) and the USA (Driscoll et al., 1999). A similar spoligotype lacking spacer 30 was also reported from a human with pulmonary BTB in Ghana (Meyer et al., 2008). These spoligotypes are members of the African 1 (Af1) clonal complex characterized by the absence of spacer 30 (Muller et al., 2009). Furthermore, the SB0944 spoligotype which is common in West Africa was reported to be the most prevalent strain in cattle in Nigeria, Chad and Cameroon (Muller et al., 2009). Our findings, based on the spoligotyping data, are thus consistent with the findings in this recently published study (Muller et al., 2009). The human isolate (hb74) with the SB0944 spoligotype presented with an ETR A-E genotype of 54543 which had also been reported in two of 59 SB0944 strains previously isolated in Nigeria (Muller et al., 2009). It is worth noting that SB1432 has only thus far been reported in cattle in Nigeria with a relatively high frequency (Muller et al., 2009). We report this SB1432 for the first time in humans (NB83) in this study. This spill over to humans may lead to its further dissemination in the human population as this strain was isolated from a patient with pulmonary tuberculosis. It was also noticed that this human isolate clustered with a group of cattle isolates showing MLVA genotypes 21-24. It presented with a similar genotype (i.e. identical ETR A-D genotype 5554) with most isolates in this cluster except a copy number of eight at Mtub02 whilst other isolates of cattle origin in the cluster had a copy number of 10 (see supplementary material).

The MLVA-16 identified $49 \mathrm{M}$. bovis isolates from the 74 strains typed and these were resolved into 30 different MLVA genotypes with a genotypic diversity of 0.97 whilst the $25 \mathrm{M}$. tuberculosis strains were resolved into 19 different MLVA 


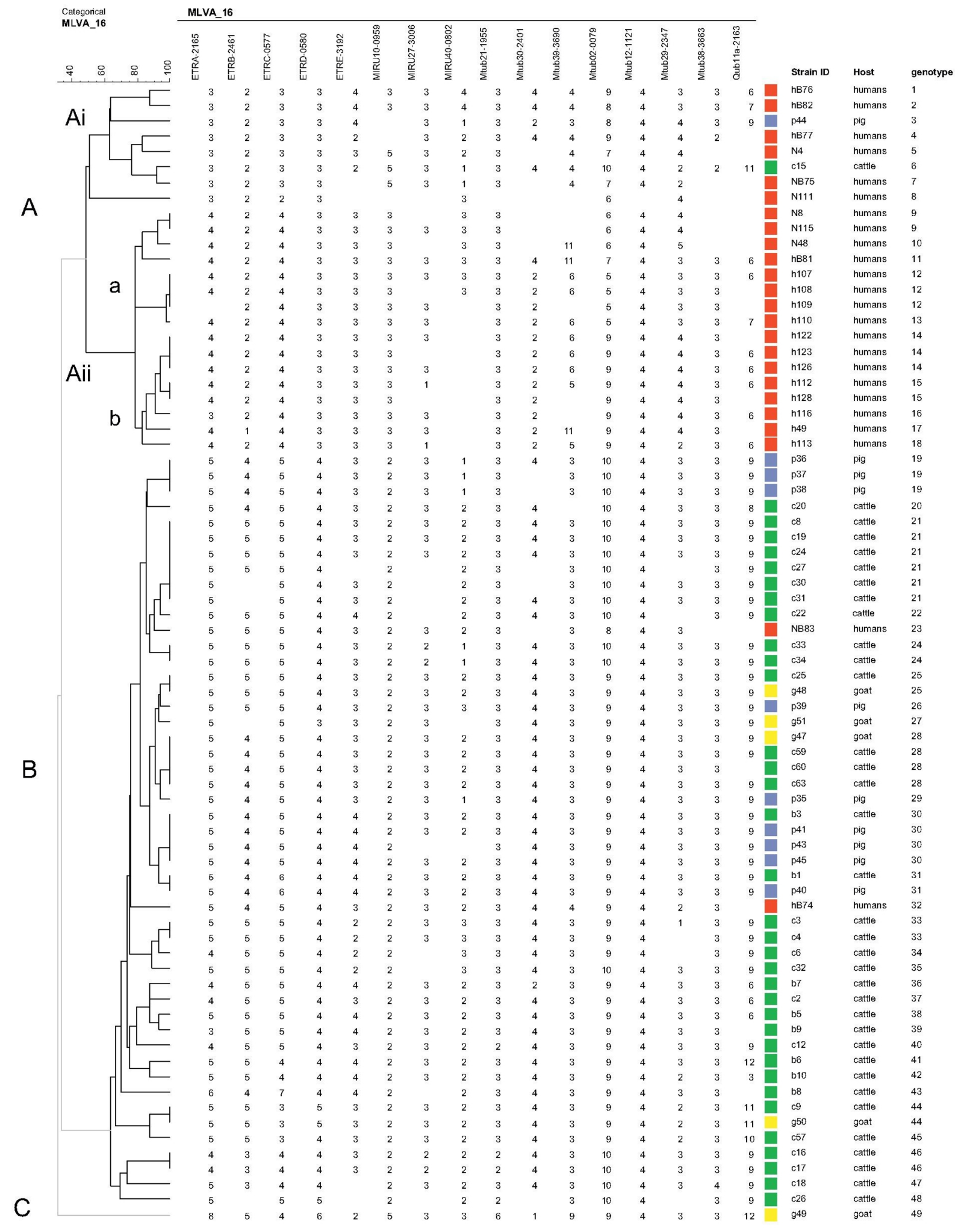

Fig. 1. Dendrogram constructed from MLVA-16 testing of $24 \mathrm{M}$. tuberculosis and $50 \mathrm{M}$. bovis strains isolated from humans and livestock in Ibadan. In the columns, the following data are presented: color code for hosts species (red: human; blue: pig; green: cattle, yellow: goat); strain identification; host and genotype. (For interpretation of the references to color in this figure legend, the reader is referred to the web version of the article.) 
genotypes. A high allelic diversity was obtained amongst the M. bovis strains from 13 of the 16 used loci. This is an indication of the level of genetic variability amongst the circulating M. bovis strains in Nigeria. The three loci which were not polymorphic in the M. bovis strains were MIRU10, Mtub12 and Mtub38. In previous studies on M. bovis strains isolated in Northern Ireland (Roring et al., 2004) and Chad (Hilty et al., 2005) MIRU10 had also been described as monomorphic. On the other hand, it was illustrated to be highly polymorphic with $\mathrm{M}$. tuberculosis isolates in this study as well as in other M. tuberculosis strains isolated from South Africa, France and the United States (Hilty et al., 2005). This study confirms that MIRU10 is of little value for the typing of M. bovis. Furthermore, it was reported by Muller et al. (2009) that the allele five was common at the ETRA locus to most M. bovis isolated previously from cattle in Nigeria. This is consistent with our data in which 31 of 49 M. bovis strains isolated from cattle including nine $\mathrm{M}$. bovis isolates from humans, goats and pigs also presented with allele five at the ETRA locus (see supplementary material). Besides allele five which was the most frequently occurring allele we also discovered alleles three, four and six from cattle isolates at the ETRA locus as was also reported by Muller et al. (2009).

Four different M. bovis MLVA genotypes were observed in different animal species. This was identified when four isolates obtained from three cattle, and one goat (c59, c60, c63, g47) had the same MLVA genotype 28 whilst strain p35 isolated from a pig showed the very closely related MLVA genotype 29, differing only at locus MIRU40. Two other strains (c25, g48) isolated from a bovine and a goat, respectively, presented with the same MLVA genotype 25 whilst strain p39 isolated from a pig showed the very closely related MLVA genotype 26 , differing only at locus MIRU40. One cattle isolate (b3) also had the identical MLVA genotype 30 with three pig isolates (p41, p43 and p45) whilst the final group with one cattle and one pig isolate (b1 and p40) also had the identical MLVA genotype 31. Another strain with a spoligopattern of SB1105, which was previously isolated from a dromedary in Chad (Diguimbaye-Djaibe et al., 2006), was also identified from a tissue sample of a female White Fulani breed of cattle (c6). This may have occurred as a result of migration of cattle and camels from Chad to cattle markets in the northern states of Nigeria.

The presence of similar strains in different host species highlights the interaction of different susceptible host species and the circulation of $\mathrm{M}$. bovis and $\mathrm{M}$. tuberculosis strains amongst them. The interaction of humans and more than one animal species in the same environment, as it occurs in the extensive system of rearing, can thus be regarded as a factor which promotes this interspecies transmission of $\mathrm{M}$. bovis and $\mathrm{M}$. tuberculosis strains.

Possible reasons for the high genetic diversity of M. bovis strains in Nigeria can be adduced to a lack of BTB control policies together with livestock exchanges with neighboring countries (Cadmus et al., 2006). The strains with the spoligopattern SB0944 were further subjected to MLVA using the ETR A-F loci and another 16 different genotypes were discovered. Whilst the approach used in the present study was not exactly the same as that of Muller et al. (2009), the diversity of M. bovis in Nigeria is however clearly reflected in both instances. All the spoligotypes identified in this study including the two new spoligotypes (SB1432 and SB1472) belonged to the Af1 clonal complex identified by the absence of spacer 30. As discussed by Muller et al. (2009), our results also suggest that the population structure of M. bovis in Nigeria is derived from a single clone lacking spacer 30. The RDAf1 deletion analysis was not carried out in this study, however, the identification of two new strains with the spacer 30 deletion supports the $\mathrm{M}$. bovis clonal population structure of $\mathrm{M}$. bovis in Nigeria, although homoplasty cannot be excluded.

This is the second time M. tuberculosis has been reported in animal isolates from Nigeria, strongly supporting the possibility of human to livestock transmission (Cadmus et al., 2006). We isolated three such strains in this study, including an isolate of the EAl clade (Brudey et al., 2006) recovered from a goat (g49). EAl strains represent the most ancestral clade of the M. tuberculosis complex (except the M. canettii ancestor) (Fabre et al., 2004). EAl strains are very characteristic in terms of spoligotype, presence of genomic regions of difference (undeleted for both TbD1 and RD9) and MLVA pattern. To the best of our knowledge, this is the first time that this type of strain has been isolated from an animal. The lack of EAI isolates from humans so far in Nigeria (Cadmus et al., 2006, this report) further suggests that it is unlikely for this goat to have been infected from a human patient or an animal source in Nigeria. In a further analysis, when this strain was compared with other strains from Africa, it clustered closely with EAl strains isolated from Sudan and the Republic of Djibouti. This suggests that this particular goat may have been infected in an East African country before being transported to Nigeria. This is an interesting finding because historically there are poor trade links between East Africa and West Africa. The isolation of this strain suggests that livestock trade between these two regions of Africa may be the source of new MTC strains in both regions. Spoligotypes of the M. tuberculosis isolates from c15 (cattle) and p44 (pig) strains were of the T and CAM families, respectively, and in a dendogram obtained from the MLVA analysis they also clustered with other members of the M. tuberculosis strains. It was also observed in the same previous study (Cadmus et al., 2006) that $69 \%$ of the M. tuberculosis isolates were of the CAM family, compared to the present observation that $56 \%$ of $\mathrm{M}$. tuberculosis strains which were spoligotyped were of the CAM family. The CAM family was also found in Burkina-Faso (Godreuil et al., 2007), Sierra Leone (Homolka et al., 2008), Niger, Ivory Coast as well as in several parts of Europe particularly France (Haddad et al., 2001) and they appear to be the dominant M. tuberculosis strain circulating in Nigeria.

Further epidemiological studies using spoligotyping and MLVA on human and livestock isolates of the MTC are recommended in Nigeria, as this will shed some light on the transmission dynamics of $M$. bovis and $M$. tuberculosis infections at the human/livestock interface. Furthermore, a careful selection of MLVA markers will give a more detailed evaluation than spoligotyping of the epidemiological relatedness between strains. The impact of cattle migration on the transmission of 
zoonotic tuberculosis is yet to be fully determined as the conditions of transport will undoubtedly have significant impacts. There is currently no nationwide molecular survey of MTC strains in Nigeria and our findings further emphasize this need.

\section{Conflict of interest statement}

The authors have no conflict of interest.

\section{Acknowledgements}

The contributions of Rob Warren and Lizma Streicher of the Division of Molecular Biology and Human Genetics, Faculty of Health Sciences, Stellenbosch University, South Africa are much appreciated. Sincere appreciation also goes to Kehinde Adesokan of the Tuberculosis Research Laboratory of the Department of Veterinary Public Health and Preventive Medicine, Faculty of Veterinary Medicine, University of Ibadan, Nigeria. Funding for this study was made available by the Department of Veterinary Tropical Diseases, Faculty of Veterinary Sciences, University of Pretoria, South Africa via a grant from the Institute of Tropical Medicine, Antwerp, Belgium and the John D. and Catherine T. MacArthur Foundation, University of Ibadan Re-entry Grant 2006.

\section{Appendix A. Supplementary data}

Supplementary data associated with this article can be found as an attachment.

\section{References}

Allix, C., Walravens, K., Saegerman, C., Godfroid, J., Supply, P., Fauville-Dufaux, M., 2006. Evaluation of the Epidemiological Relevance of Variable-Number Tandem-Repeat Genotyping of Mycobacterium bovis and Comparison of the Method with IS6110 Restriction Fragment Length Polymorphism Analysis and Spoligotyping. J. Clin. Microbiol. 44, 1951-1962.

Ayele, W.Y., Neill, S.D., Zinsstag, J., Weiss, M.G., Pavlik, I., 2004. Bovine tuberculosis: an old disease but a new threat to Africa. Int. J. Tuberc. Lung Dis. 8, 924-937.

Brudey, K., Driscoll, J.R., Rigouts, L., Prodinger, W.M., Gori, A., Al-Hajoj, S.A., Allix, C., Aristimuño, L., Arora, J., Baumanis, V., Binder, L., Cafrune, P., Cataldi, A., Cheong, S., Diel, R., Ellermeier, C., Evans, J.T., Fauville-Dufaux, M., Ferdinand, S., Garcia de Viedma, D., Garzelli, C., Gazzola, L., Gomes, H.M., Cristina Guttierez, M., Hawkey, P.M., van Helden, P.D., Kadival, G.V., Kreiswirth, B.N., Kremer, K., Kubin, M., Kulkarni, S.P., Liens, B., Lillebaek, T., Minh Ly, H., Martin, C., Martin, C., Mokrousov, I., Narvskaïa, O., Fong Ngeow, Y., Naumann, L., Niemann, S., Parwati, I., Rahim, Z., Rasolofo-Razanamparany, V., Rasolonavalona, T., Lucia Rossetti, M., Rüsch-Gerdes, S., Sajduda, A., Samper, S., Shemyakin, I.G., Singh, U.B., Somoskovi, A., Skuce, R.A., van Soolingen, D., Streicher, E.M., Suffys, P.N., Tortoli, E., Tracevska, T., Vincent, V., Victor, T.C., Warren, R.M., Fan Yap, S., Zaman, K., Portaels, F., Rastogi, N., Sola, C., 2006. Mycobacterium tuberculosis complex genetic diversity: mining the fourth international spoligotyping database (SpolDB4) for classification, population genetics and epidemiology. BMC Microbiol. 6, 23.

Cadmus, S., Palmer, S., Okker, M., Dale, J., Gover, K., Smith, N., Jahans, K., Hewinson, R.G., Gordon, S.V., 2006. Molecular analysis of human and bovine tubercle bacilli from a local setting in Nigeria. J. Clin. Microbiol. 44, 29-34.

Diguimbaye-Djaibe, C., Hilty, M., Ngandolo, R., Mahamat, H.H., Pfyffer, G.E., Baggi, F., Hewinson, G., Tanner, M., Zinsstag, J., Schelling, E., 2006. Mycobacterium bovis isolates from tuberculous lesions in Chadian zebu carcasses. Emerg. Infect. Dis. 12, 769-771.

Driscoll, J.R., McGarry, M.A., Taber, H.W., 1999. DNA typing of a nonviable culture of Mycobacterium tuberculosis in a homeless shelter outbreak. J. Clin. Microbiol. 37, 274-275.

Fabre, M., Koeck, J.L., Le Fleche, P., Simon, F., Herve, V., Vergnaud, G.P.C., 2004.High genetic diversity revealed by variablenumber tandem repeat genotyping and analysis of hsp65 gene polymorphism in a large collection of "Mycobacterium Canettii" strains indicates that the M. tuberculosis complex is a recently emerged clone of "M. Canettii" J. Clin. Microbiol. 42, 32483255.

Godreuil, S., Torrea, G., Terru, D., Chevenet, F., Diagbouga, S., Supply, P., Van de Perre, P., Carriere, C., Banuls, A.L., 2007. First molecular epidemiology study of Mycobacterium tuberculosis in Burkina Faso. J. Clin. Microbiol. 45, 921-927.

Haddad, N., Ostyn, A., Karoui, C., Masselot, M., Thorel, M.F., Hughes, S.L., Inwald, J., Hewinson, R.G., Durand, B., 2001. Spoligotype diversity of Mycobacterium bovis strains isolated in France from 1979 to 2000. J. Clin. Microbiol. 39, 3623-3632.

Hilty, M., Diguimbaye, C., Schelling, E., Baggi, F., Tanner, M., Zinsstag, J., 2005. Evaluation of the discriminatory power of variable number tandem repeat (VNTR) typing of Mycobacterium bovis strains. Vet. Microbiol. 109, $217-222$.

Homolka, S., Post, E., Oberhauser, B., George, A.G., Westman, L., Dafae, F., Rusch-Gerdes, S., Niemann, S., 2008. High genetic diversity among Mycobacterium tuberculosis complex strains from Sierra Leone. BMC Microbiol. 8, 103.

Hunter, P.R., Gaston, M.A., 1988. Numerical index of the discriminatory ability of typing systems: an application of Simpson's index of diversity. J. Clin. Microbiol. 26, 2465-2466. 
Idigbe, E.O., Anyiwo, C.E., Onwujekwe, D.I., 1986. Human pulmonary infections with bovine and atypical mycobacteria in Lagos. Niger. J. Trop. Med. Hyg. 89, 143-148.

Jenkins, A.O., Venter, E.H., Hutamo, K., Godfroid, J., 2010. Comparison of the capillary and agarose electrophoresis based multiple locus VNTR (variable number of tandem repeats) analysis (MLVA) on Mycobacterium bovis isolates. Vet. Microbiol., doi:10.1016/i.vet-mic.2010.02.036.

Kamerbeek, J., Schouls, L., Kolk, A., van Agterveld, M., van Soolingen, D., Kuijper, S., Bunschoten, A., Molhuizen, H., Shaw, R., Goyal, M., van Embden, J., 1997. Simultaneous detection and strain differentiation of Mycobacterium tuberculosis for diagnosis and epidemiology. J. Clin. Microbiol. 35, 907-914.

Le Fléche, P., Fabre, M., Denoeud, F., Koeck, J.L., Vergnaud, G., 2002. High resolution, on-line identification of strains from the Mycobacterium tuberculosis complex based on tandem repeat typing. BMC Microbiol. 2, 37.

Mawak, J., Gomwalk, N., Bello, C., Kandakai-Olukemi, Y., 2006. Human pulmonary infections with bovine and environment (atypical) mycobacteria in Jos, Nigeria. Ghana Med. J. 40, 132-136.

Meyer, C.G., Scarisbrick, G., Niemann, S., Browne, E.N.L., Chinbuah, M.A., Gyapong, J., Osei, I., Owusu-Dabo, E., Kubica, T., Rüsch-Gerdes, S., Thye, T., Horstmann, R.D., 2008. Pulmonary tuberculosis: virulence of Mycobacterium africanum and relevance in HIV co-infection. Tuberculosis $88,482-489$.

Muller, B., Steiner, B., Bonfoh, B., Fane, A., Smith, N.H., Zinsstag, J., 2008. Molecular characterisation of Mycobacterium bovis isolated from cattle slaughtered at the Bamako abattoir in Mali. BMC Vet. Res. 4, 26.

Muller, B., Hilty, M., Berg, S., Garcia-Pelayo, M.C., Dale, J., Boschiroli, M.L., Cadmus, S., Ngandolo, B.N., Godreuil, S., DiguimbayeDjaibe, C., Kazwala, R., Bonfoh, B., Njanpop-Lafourcade, B.M., Sahraoui, N., Guetarni, D., Aseffa, A., Mekonnen, M.H., Razanamparany, V.R., Ramarokoto, H., Djonne, B., Oloya, J., Machado, A., Mucavele, C., Skjerve, E., Portaels, F., Rigouts, L., Michel, A., Muller, A., Kallenius, G., van Helden, P.D., Hewinson, R.G., Zinsstag, J., Gordon, S.V., Smith, N.H., 2009. African 1, an epidemiologically important clonal complex of Mycobacterium bovis dominant in Mali, Nigeria, Cameroon, and Chad. J. Bacteriol. 191, 1951-1960.

Njanpop-Lafourcade, B.M., Inwald, J., Ostyn, A., Durand, B., Hughes, S., Thorel, M.F., Hewinson, G., Haddad, N., 2001. Molecular typing of Mycobacterium bovis isolates from Cameroon. J. Clin. Microbiol. 39, 222-227.

Ocepek, M., Pate, M., Zolnir-Dovc, M., Poljak, M., 2005. Transmission of Mycobacterium tuberculosis from human to cattle. J. Clin. Microbiol. 43, 3555-3557.

Roring, S., Scott, A.N., Glyn Hewinson, R., Neill, S.D., Skuce, R.A., 2004. Evaluation of variable number tandem repeat (VNTR) loci in molecular typing of Mycobacterium bovis isolates from Ireland. Vet. Micro-biol. 101, 65-73.

Salami, A.K., Katibi, I.A., 2007. Tuberculosis among the health-care workers of the university of Ilorin teaching hospital. Trop. Doct. 37, 251

Salami, A.K., Katibi, I.A., 2006. Human immunodeficiency virus-associated tuberculosis: pattern and trend in the university of Ilorin teaching hospital. Afr. J. Med. Med. Sci. 35, 457-460.

Srivastava, K., Chauhan, D.S., Gupta, P., Singh, H.B., Sharma, V.D. Yadav, V.S., Sreekumaran, Thakral, S.S., Dharamdheeran, J.S Nigam, P., Prasad, H.K., Katoch, V.M., 2008. Isolation of Mycobacterium bovis and M. tuberculosis from cattle of some farms in North India-possible relevance in human health. Indian J. Med. Res. 128, 26-31.

Van Soolingen, D., 2001. Molecular epidemiology of tuberculosis and other mycobacterial infections: main methodologies and achievements. J. Intern. Med. 249, 1-26.

Warren, R.M., Gey van Pittius, N.C., Barnard, M., Hesseling, A., Engelke, E., de Kock, M., Gutierrez, M.C., Chege, G.K., Victor, T.C., Hoal, E.G., van Helden, P.D., 2006. Differentiation of Mycobacterium tuberculosis complex by PCR amplification of genomic regions of difference. Int. J. Tuberc. Lung Dis. 10, 818-822. 